\title{
Aprendizaje Integrado de Ciencias Sociales e Inglés por Medio de una Unidad Didáctica Basada en la Geografía Local
}

\author{
Integrated Learning of Social Sciences and English through a Didactic Unit Based on \\ Local Geography
}

Victor Manuel Jaimes-Molina ${ }^{1}$

\section{○ EDICIÓN: @E-CIVTAC}

Recibido: 25/junio/2021

Aceptado: $18 /$ septiembre/2021

Publicado: 24/septiembre/2021

我 País

${ }^{1}$ Colombia

\section{IIIII Institución}

${ }^{1}$ Universidad de Pamplona

\section{CCorreo Eletrónico \\ ${ }^{1}$ victormanueljaimes@gmail.com}

\section{(iD) ORCID}

'https://orcid.org/0000-0002-4300-8956

\section{Citar así: 4 APA / IEEE}

Jaimes-Molina, V. (2021). Aprendizaje Integrado de Ciencias Sociales e Inglés por Medio de una Unidad Didáctica Basada en la Geografía Local. Revista TecnológicaEducativa Docentes 2.0, 11(2), 66-75. https://doi.org/10.37843/rted.v11i2.254

\section{Resumen}

La comunicación e interacción social son la razón de ser del uso de un idioma, por lo tanto, la enseñanza de lenguas debe privilegiar las habilidades relacionadas con la producción oral y la comprensión auditiva. Esta investigación contrasta la dificultad en la enseñanza y aprendizaje del inglés desde un enfoque comunicativo con el aprendizaje integrado de contenidos y lenguas (CLIL). Dicho contraste se dio por medio del diseño de una unidad didáctica de ciencias sociales, con el propósito de analizar la incidencia de CLIL en estudiantes de 12 a 14 años en la Institución José Odel Lizarazo. Para esto, el enfoque se llevó a cabo desde un paradigma cualitativo, proponiendo que la unidad didáctica partiera de la geografía local del departamento de Arauca y con las consideraciones del enfoque basado en tareas. El análisis se realizó por medio de la observación, notas de campo, registro audiovisual, cuestionario de entrada de salida, lo cual permitió comprender que un buen desempeño en la habilidad de "listening" propició la producción oral. Por otra parte, la integración de contenidos de ciencias sociales e inglés favoreció un aprendizaje activo en los estudiantes. Finalmente, el CLIL ofreció una alternativa a la necesidad lingüística de los estudiantes.

Palabras clave: Aprendizaje, ciencias sociales, inglés, integración.

\section{Abstract}

Communication and social interaction are the reason for language use; therefore, language teaching should privilege skills related to oral production and listening comprehension. This research contrasts the difficulty of teaching and learning English from a communicative approach with content and language integrated learning (CLIL). This contrast was made by designing a didactic unit of social sciences to analyze the incidence of CLIL in students from 12 to 14 years old from the José Odel Lizarazo Institution. For this purpose, the approach was carried out from a qualitative paradigm, proposing that the didactic unit be based on the local geography of the department of Arauca and with the considerations of the task-based approach. The analysis utilized observation, field notes, audiovisual recording, and the input-output questionnaire, which made it possible to understand that a good performance in the skill "listening" favored oral production. On the other hand, the students' integration of social science and English content tended to active learning. Finally, CLIL offered an alternative to the linguistic needs of the students.

Keywords: Learning, social studies, english, integration.
V. Jaimes-Molina, "Aprendizaje Integrado de Ciencias Sociales e Inglés por Medio de una Unidad Didáctica Basada en la Geografía Local", RTED, vol. 11, n. ${ }^{\circ} 2$, pp. 66-75, sep. 2021. 


\section{Introducción}

La comunicación e interacción social son la razón de ser del uso de un idioma, por lo tanto, la enseñanza de lenguas debe privilegiar las habilidades relacionadas con la producción oral y la comprensión auditiva Chenlu (2015), esto contrasta con los enfoques tradicionales con los que se ha abordado la enseñanza en las instituciones públicas de Colombia, América Latina y el mundo, en las que se requiere de la repetición de fórmulas, reglas, disciplina mental, memorización de vocabulario, análisis gramatical, un alto nivel intelectual y concentración Marsh (2002). No obstante, estos métodos han mostrado que los ciudadanos del mundo no adquieren las competencias lingüísticas necesarias que se requiere para vivir en una dinámica de integración y globalización, de hecho, pruebas como el ICFES, muestran que en Colombia el $50 \%$ de los estudiantes del grado once ni siquiera alcanzan el nivel A1, el más bajo según el marco común europeo (ICFES, 2019).

Una situación semejante ocurre en la Institución José Odel Lizarazo en Saravena Colombia con el Proyecto Educativo Institucional (PIE) (2012), donde se hizo una intervención en el aula con el aprendizaje integrado de contenido y lengua (CLIL), este nace en la unión europea, como respuesta al proceso de integración que requería que los ciudadanos se comunicaran por lo menos en un idioma adicional al de su lengua materna. Marsh define el aprendizaje integrado de contenido y lenguaje (CLIL) como "un término genérico semejante a una sobrilla que abarca cualquier actividad en la cual se usa un idioma extranjero como herramienta para el aprendizaje de una materia no lingüística" $(2002,58)$, de modo que esa materia como el idioma extranjero tengan un rol curricular en conjunto, CLIL promueve que el idioma sea una herramienta por medio de la cual se enseñen contenidos de distintas asignaturas, pero, se diferencia de los demás enfoques en que tiene tres dimensiones que son los conceptos que son los contenidos de la materia no lingüística, el lenguaje y los procedimientos que enmarcan la forma en cómo se va abordar la enseñanza.

Para esta última dimensión, se apela al enfoque basado en tareas, según Nunan (2004) una tarea en términos pedagógicos es una actividad o acción que es llevada a cabo como el resultado de un procedimiento o del entendimiento del lenguaje. Por ejemplo, dibujar un mapa mientras escucha una grabación, escuchar una instrucción, o desempeñar una orden puede ser referida como tarea lleva a que la atención del estudiante esté enfocada en la actividad que tiene que desarrollar así que éste ya no va a estar estresado por la parte lingüística la cuál pasa a un segundo plano, y para alcanzar esto, Nunan (2004) recomienda que las actividades tengan una secuencia, en la que se comience con lecciones sencillas y se vaya progresando, utilizando el principio del reciclaje, que consiste en repetir los aspectos claves de una lecciones anteriores.

Luego, de analizar la base teórica del aprendizaje integrado de contenido y lenguas, se hizo una propuesta pedagógica, que buscara analizar la incidencia de la metodología CLIL en jóvenes de 12 a 14 años, todo esto a partir, del diseño $\mathrm{e}$ implementación de una unidad didáctica de ciencias sociales que se implementó durante siete sesiones, esta unidad se construyó desde la geografía local en el municipio de Saravena y de la experiencia cotidiana de las familias de los estudiantes. De acuerdo con lo anterior, se consideró pertinente, diseñar, implementar y evaluar una unidad didáctica que integrara las ciencias sociales con el idioma inglés por medio de la geografía local y aplicando el aprendizaje integrado de contenidos y lengua.

\section{Metodología}

Esta investigación se realizó bajo un paradigma cualitativo, puesto que el trabajo desarrollado, la recolección de datos y los resultados esperados fueron analizados bajo la observación de la relación docente estudiante. De acuerdo con lo mencionado por Bisquerra la metodología cualitativa "se refiere a aquellos procedimientos de investigación que proporcionan datos descriptivos $\mathrm{y}$ 
funcionalmente relevantes, contextualizándolos en el ámbito social donde ocurren naturalmente" (2004, p.82) y que permite estudiar los fenómenos más complejos dando un alto énfasis a la reflexión, además que este tipo de investigación le apunta a comprender la realidad para transformarla. En armonía con lo anterior, este proyecto no se detiene en el diagnóstico de un problema, y el análisis de sus posibles causas, pues éste, contrasta la realidad con la metodología de aprendizaje integrado de contenido y lenguas de Marsh (2002), en busca de validar de qué manera esta alternativa ofrece una solución a la problemática.

Por otra parte, la esencia del objeto de estudio es analizar el porqué de las cosas, ofreciendo una descripción y una explicación de la realidad, de acuerdo con la experiencia del investigador con los hechos (Marín, 2018). Es preciso mencionar que, la metodología CLIL, en particular, tiene unas características que fueron asimiladas de otros enfoques predecesores; uno de estos enfoques, conocido como el método directo afirma que el aprendizaje de un idioma se da de forma acelerada cuando se da en un contexto semejante, al de un niño que aprende ese idioma en el país cuya lengua materna sea ese idioma (Howatt, 1984); en este sentido, los encuentros presenciales con los estudiantes simulan un ambiente de aprendizaje semejante al de un país de habla inglesa.

Para esto, se seleccionó una población de cinco estudiantes residentes en el área rural de Saravena, del grado séptimo en la Institución José Odel Lizarazo, estos estudiantes están entre los 12 y 14 años de edad, y pertenecen a un contexto familiar con dificultades socioeconómicas. Estos estudiantes, manifestaron afinidad gusto por el idioma y sus familias mostraron disponibilidad a participar en la unidad didáctica, la cual requería de trabajo presencial en las instalaciones de la institución, siguiendo los pasos de la investigación acción-participante planteadas por Álvarez \& Álvarez:
1. Delimitación del problema a investigar en la acción.

2. Planificación del proceso de acción.

3. Ejecución de la acción.

4. Evaluación de lo generado en la acción.

5. Revisar con base en la evaluación del plan los resultados para diseñar uno nuevo.

6. Revisar y reelaborar el plan tantas veces como sea necesario (2014, p. 23).

El plan de acción dio inicio con una prueba diagnóstica que tenía por objeto evidenciar las habilidades de los estudiantes, de acuerdo con el nivel A2 del marco común europeo, dicha prueba fue diseñada siguiendo la estructura del examen "Key english test" y se aplicó de forma presencial, dicha prueba, arrojó información para hacer algunos ajustes a la unidad didáctica, la cual había sido planeada conforme a los derechos básicos de aprendizaje propuestos por el Ministerio de Educación (2019) en Colombia, esta, se implementó en siete sesiones, en las que se integraron las ciencias sociales y el inglés por medio de la versión "soft" de CLIL en aquellos puntos en que convergieron. Los resultados de las pruebas diagnóstica y final se acompañó de datos cuantitativos, sin embargo, en la categorización y análisis de las preguntas prevaleció la esencia cualitativa.

Es importante mencionar que CLIL tiene unos niveles de intensidad, que van desde lo denominado como "soft" CLIL que consiste en clases de idiomas donde el docente planea la enseñanza de idiomas en torno a una serie de temas, hasta la versión "hard", (Ball, Clegg \& Kelly, 2015). CLIL que se trata de un proceso de enseñanza completamente en inglés simulando un entorno nativo, esta unidad, organizó sesiones de trabajo en torno a las ciencias sociales transversalizados por el idioma inglés, y para analizar estos datos se hizo uso de un registro de notas. La observación según Tamayo (2004) consiste en recolectar, registrar y reflexionar en torno a los datos de manera sistemática, lo que permite una revisión clara, objetiva de los hechos, y de la categorización de los datos, teniendo en cuenta que tanto las principales 
variables como las subcategorías se desprenden de las tres dimensiones de CLIL.

De manera, que la dimensión del lenguaje permite observar el desempeño de la producción oral y la comprensión auditiva, la dimensión del contenido evidencia la pertenencia territorial y la apropiación del contenido de ciencias sociales, y la dimensión de procedimientos permite observar el desempeño de los estudiantes en la realización de actividades, la interacción social y el uso del idioma para informar respecto a las ciencias sociales.

\section{Resultados}

El diseño de la unidad didáctica requirió del uso de los conocimientos empíricos de los estudiantes por su territorio, los cuáles fueron adaptados a los estándares curriculares de ciencias sociales. Estos conocimientos, se organizaron de manera secuencial, para integrar el idioma inglés en los puntos en que podían converger, sin embargo, el uso CLIL, implicó sacrificios tanto en la ciencias sociales, como en la parte lingüística, lo que significó la selección de contenidos básicos que en realidad se enseñan en el grado quinto, como lo es el DBA número 1 propuesto por el Ministerio de Educación Nacional (MEN) en la guía 6 en la que demanda que los estudiantes deben comprender la organización territorial existente en Colombia y las particularidades geográficas de las regiones $(2004$, p. 25)

En cuanto a los lineamientos curriculares lingüísticos, la guía 22 del MEN (2006) en Colombia sugiere que los estudiantes del grado cuarto a séptimo deberían, tener un desempeño equivalente al nivel A1 de acuerdo con el marco común europeo. De esta forma, tanto la guía número 6 , como la 22 , ofrecieron el soporte curricular sobre la cual se diseñó una propuesta de unidad didáctica con sesiones secuenciales, que integra las ciencias sociales y el inglés (ver Tabla 1-5).

\section{Tabla 1}

\section{Colombia y sus vecinos 90 minutos}

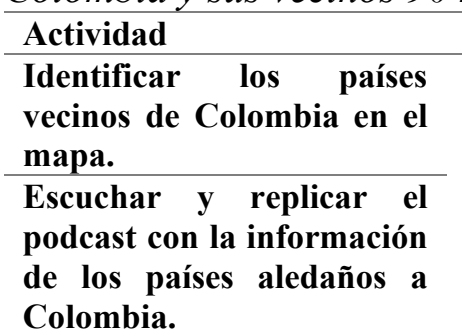

Secuencias y fases

\section{Contenidos y objetivos.}

Uso del mapa político de América, para localizar a Colombia y sus vecinos de forma auditiva y oral mediante la aplicación de preposiciones.

\section{Gramática}

Prepositions

- Next to

- On the left

- On the right

- Between

- In the north

- In the south

1) Inicio: el sistema solar, el planeta tierra.

2) Desarrollo: ¿Qué son los continentes?, ¿qué son los países?, Colombia en su vecindario.

3) Experimentación: utilizar las preposiciones para localizar territorios en un mapa.

Comprender las razones principales por las que se dividen los territorios.

4) Síntesis y cierre: El estudiante elabora un croquis de la finca donde vive y ubica sitios de referencia.

Nota. Evidencia de aprendizaje. Relaciona su izquierda, derecha, adelante, atrás en relación con los puntos cardinales por medio de las preposiciones al ubicar los países limítrofes con Colombia, elaboración propia (2021). 


\section{Tabla 2}

División geográfica de los territorios 90 Minutos

\section{Actividad \\ Contenidos y objetivos.}

Identificar los detalles

relevantes en la geografía

física de Saravena.

Realizar comparaciones de

manera oral y escrita entre

los departamentos de

Colombia, su tamaño y su temperatura.
Compara diferentes características de los

departamentos de Colombia en el idioma inglés usando adjetivos sencillos como grande, pequeño, frío, pequeño.

Identifica el lugar donde reside cada estudiante con sus características.

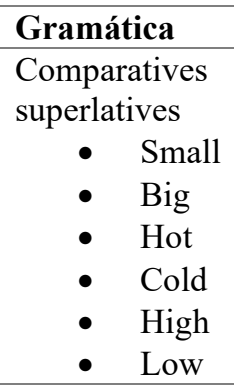

1) Inicio: empalme con síntesis y generalización de la sesión 1, repaso de definición de departamento, continente, país.

2) Desarrollo: Características físicas del municipio de Saravena, lugares montañosos, lugares llanos, ubicación de las diferentes veredas.

3) Síntesis y cierre: Utilizar los adjetivos comparativos para describir los lugares donde residen los estudiantes. Cada estudiante realiza el mapa de su territorio y describe las características más importantes.

Nota. Evidencia de aprendizaje. Diferencia la organización política del país a partir de comparaciones de la extensión territorial de los departamentos por medio de adjetivos comparativos en el idioma inglés, elaboración propia (2021).

\section{Tabla 3}

Demografia y temperatura del departamento de Arauca 90 Minutos

\begin{tabular}{|c|c|c|}
\hline Actividad & Contenidos y objetivos. & Gramática \\
\hline $\begin{array}{l}\text { Contrastar la frecuencia de } \\
\text { lluvias en los distintos } \\
\text { departamentos de Colombia } \\
\text { por medio de oraciones que } \\
\text { utilicen los adverbios de } \\
\text { frecuencia }\end{array}$ & $\begin{array}{l}\text { Emplea adverbios de frecuencia para identificar } \\
\text { los departamentos más lluviosos del país, lo más } \\
\text { secos y lo más secos. } \\
\text { Comprende la variación con respecto a la altitud de } \\
\text { la tierra sobre el nivel del mar. } \\
\text { Compara los picos de Colombia con usando los } \\
\text { adjetivos low y high. }\end{array}$ & $\begin{array}{l}\text { Vocabulario relati } \\
\text { clima. Uso de verb } \\
\text { personales. } \\
\text { - Foggy } \\
\text { - Stormy } \\
\text { - Sunny } \\
\text { - Rainy } \\
\text { - Windy } \\
\text { - There is } \\
\text { - There are }\end{array}$ \\
\hline
\end{tabular}

Nota. Evidencia de aprendizaje. Relaciona la existencia en la temperatura del departamento de Arauca de acuerdo con los diferentes pisos térmicos, elaboración propia (2021).

\section{Tabla 4}

Actividades económicas y cotidianas del departamento 90 Minutos

\begin{tabular}{lll}
\hline Actividad & Contenidos y objetivos. & Gramática \\
\hline $\begin{array}{l}\text { Emplea vocabulario en } \\
\text { inglés para hablar de las }\end{array}$ Principales actividades económicas del área & Vocabulario en contesto \\
$\begin{array}{l}\text { actividades económicas de } \\
\text { sus familias. }\end{array}$ & Cocoa \\
\hline $\begin{array}{l}\text { Relata las jornadas de } \\
\text { laboreo que realizan los }\end{array}$ & Yucca \\
padres y cuidadores en las & Cheese \\
fincas del territorio. & Animals. \\
\end{tabular}

Nota. Evidencia de aprendizaje. Plantea situaciones que le permiten reconocer la importancia que tienen las actividades económicas en el municipio de Saravena, elaboración propia (2021). 
Tabla 5

Lugares importantes en Saravena. 90 Minutos

Actividad

Identifica los lugares donde puede acceder a bienes básicos como supermercado, veterinaria, farmacia, tienda de ropa.

Realiza preguntas de forma colaborativa, indagando por el precio de diversos productos necesarios para la canasta básica.

Contenidos y objetivos.

Bienes y servicios de uso fundamental en los moradores del municipio.

Produce contenido oral sencillo en el idioma inglés, utilizando las preposiciones de lugar, los adjetivos comparativos y superlativos para describir las características principales del territorio en el departamento de Arauca.

\section{Gramática}

Prepositions

- How much

- It costs.

- In the north

- In the south

Comparatives

superlatives

- Small

- $\mathrm{Big}$

- Hot

- Cold

\section{Secuencia y fases}

1) Inicio: Ejercicio de retroalimentación aplicando el principio del reciclaje en las actividades de listening, esta actividad a la vez sirvió de empalme con los contenidos de la sesión 6 .

2) Desarrollo: Lugares donde se compran los distintos bienes y servicios que requiere un campesino.

3) Síntesis y cierre: Trabajo colaborativo en el que los estudiantes formulan y responden preguntas en las que se da razón de los precios de los principales productos que consume un habitante de la región.

Nota. Evidencia de aprendizaje. Propone situaciones en las que da soluciones a necesidades básicas a partir de los bienes y servicios disponibles en el entorno, elaboración propia (2021).

Una vez, que se finalizó con la etapa de diseño, se procedió a realizar una prueba que diagnosticó el nivel de los estudiantes en las habilidades de "listening" y "speaking". La primera sección consistió en cinco preguntas, las cuáles fueron diseñadas teniendo en cuenta las dimensiones de CLIL, estas dimensiones constituyen además las categorías principales de la teoría, Corbin \& Strauss mencionan que "las categorías son conceptos que se derivan de los datos, en orden de ser analizados y estudiados de forma óptima" (2002, p. 124)
La sección de "listening" tuvo ocho preguntas (ver Tabla 6), cada una con ocho enunciados en inglés que se reproducían tres veces, con base en los enunciados respondían las preguntas, las cuales tenían tres opciones de respuesta, pero solo una opción correcta, los resultados fueron analizados de forma descriptiva, sin embargo, la siguiente tabla los muestra unos porcentajes que tienen el propósito de dar una idea más sencilla del desempeño de los estudiantes.

\section{Tabla 6}

Desempeño en la habilidad de" listening”

\section{PRUEBA DIAGNÓSTICA}

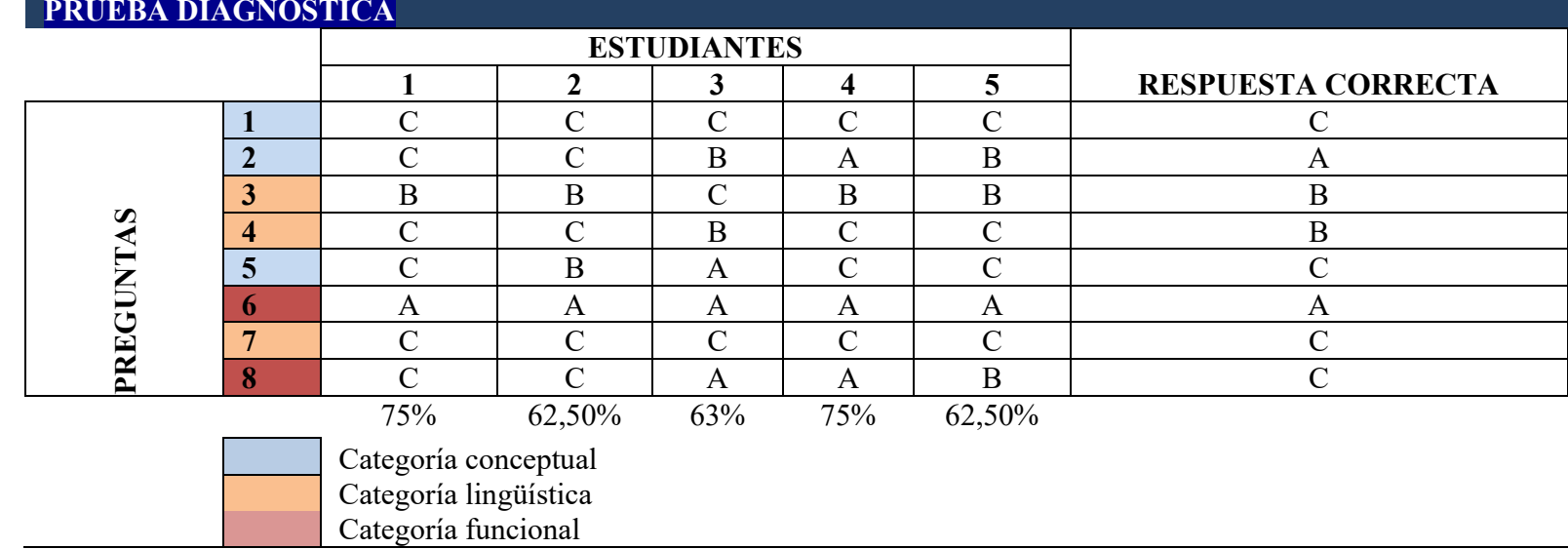

Nota. Dimensiones expresadas en porcentaje, elaboración propia (2021). 
La siguiente sección del diagnóstico fue la prueba de speaking (ver Tabla 7), los parámetros que se tuvieron en cuenta, para el análisis de esta sección fueron la claridad al expresarse, el uso correcto del lenguaje, y el desarrollo del tema. Dos estudiantes se sintieron abrumados ante la experiencia de tener que comunicarse únicamente en inglés, ellos no pudieron responder a ninguna de las preguntas. Finalmente, una de las estudiantes mostró que comprendía algunas de las palabras al momento de oír las oraciones y realizó intentos por responder, pero no consiguió dar un desarrollo claro a lo que se le estaba preguntando.

Tabla 7

Desempeño en la habilidad de speaking

\begin{tabular}{llllll}
\hline \multicolumn{7}{l}{ ESTUDIANTES } & & & \\
& $\mathbf{1}$ & $\mathbf{2}$ & $\mathbf{3}$ & $\mathbf{4}$ & $\mathbf{5}$ \\
\hline CLARIDAD & $55 \%$ & $65 \%$ & $25 \%$ & $\mathrm{NR}$ & $\mathrm{NR}$ \\
\hline $\begin{array}{l}\text { USO DEL } \\
\text { LENGUAJE }\end{array}$ & $60 \%$ & $70 \%$ & $20 \%$ & $\mathrm{NR}$ & $\mathrm{NR}$ \\
$\begin{array}{l}\text { DESARROLLO } \\
\text { DEL TEMA }\end{array}$ & $65 \%$ & $65 \%$ & $25 \%$ & $\mathrm{NR}$ & $\mathrm{NR}$
\end{tabular}

Nota. Prueba diagnóstica sección de speaking, elaboración propia (2021).

Los resultados de la implementación, fueron analizados por medio de la observación, según Hernández-Sampieri, Fernández-Collado \& Baptista-Lucio, ésta "no debe ser es mera contemplación (sentarse a ver el mundo y tomar notas); implica adentrarnos profundamente en situaciones sociales y mantener un papel activo, así como una reflexión permanente" (2014, p. 399), para esto, en cada sesión hubo un registro de notas en el que se estuvo atento a los aspectos metodológicos de CLIL, pero también notas descriptivas e inferenciales, privilegiando constantemente la reflexión sobre lo que sucedió en el aula.

Durante las sesiones implementación, los jóvenes mostraron agrado, voluntad por participar, y fueron capaces de reproducir frases sencillas, en las que utilizaron el idioma inglés para hablar de su territorio, pero, tuvieron dificultades con la habilidad de "listening", específicamente cuando las actividades consistían en extraer información de un audio, ellos tuvieron dificultad con identificar números en el orden de las centenas de mil para el registro de datos demográficos, por lo tanto, fue necesario realizar adaptaciones a las actividades propuestas, y ajustar el nivel de complejidad, sin embargo, fue indispensable, fortalecer esta habilidad, según Krashen citado por
Contreras (2012), que hay una relación directa entre lo que una persona lee y escucha, con lo que ésta escribe o habla (p.124). Por lo tanto, la habilidad de "listening" es un prerrequisito que antecede a la de "speaking".

Para los últimos dos encuentros, los estudiantes se concentraron en construir diálogos entre ellos, que involucraran los aspectos que habían abordado, tales como la compra de bienes y servicios en el municipio de Saravena, la locación de sus veredas en la zona rural, el precio de algunos productos, $\mathrm{y}$ pese a que dos de los estudiantes mantenían dificultades con la producción oral, el trabajo colaborativo permitió que todos tuvieran mejoras significativas en dicha habilidad.

La aplicación de la unidad didáctica de ciencias sociales, integrando el idioma inglés, favoreció el desarrollo de las habilidades orales, en los estudiantes que participaron, pero para que esto pudiera ocurrir, se hizo necesario fortalecer la capacidad de escucha en los estudiantes (ver Tabla 8), como consecuencia, esta metodología ayudó a aprender y afianzar vocabulario en inglés ya que el aprendizaje ocurre en contexto, así mismo, aquellos contenidos alusivos a la geografía y característica de la región resultaron bastante significativos, pues los estudiantes evidenciaron apropiación por el conocimiento de su territorio. 
Al finalizar la implementación de la unidad didáctica, los estudiantes rindieron una prueba con estructura semejante al diagnóstico y analizada bajo las mismas categorías que emanan de la teoría principal.

Tabla 8

Sección de listening

\begin{tabular}{|c|c|c|c|c|c|c|c|}
\hline \multicolumn{7}{|c|}{ ESTUDIANTES } & \multirow{2}{*}{$\begin{array}{l}\text { RESPUESTA } \\
\text { CORRECTA }\end{array}$} \\
\hline & & 1 & 2 & 3 & 4 & 5 & \\
\hline \multirow{4}{*}{ 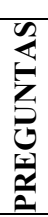 } & 1 & B & B & B & B & B & B \\
\hline & 2 & A & $\mathrm{A}$ & $\mathrm{C}$ & A & $\mathrm{C}$ & $\mathrm{A}$ \\
\hline & 3 & $\mathrm{C}$ & $\mathrm{C}$ & A & $\mathrm{C}$ & $\mathrm{C}$ & $\mathrm{C}$ \\
\hline & 4 & A & $\mathrm{A}$ & A & A & $\mathrm{C}$ & $\mathrm{A}$ \\
\hline & 5 & B & B & B & A & B & B \\
\hline & 6 & B & B & B & B & B & B \\
\hline & 7 & $\mathrm{C}$ & $\mathrm{C}$ & $\mathrm{C}$ & $\mathrm{C}$ & $\mathrm{C}$ & $\mathrm{C}$ \\
\hline & 8 & B & $\mathrm{C}$ & B & A & $\mathrm{C}$ & $\mathrm{C}$ \\
\hline
\end{tabular}

Después de la implementación de la unidad didáctica los estudiantes mostraron mejoras tanto en listening como en speaking (ver Tabla 9) en la prueba dentro del contexto de las tres categorías que están relacionadas con el entorno geográfico, sin embargo, las mejoras más significativas se evidenciaron en esta última habilidad, teniendo en cuenta que para el diagnóstico dos de los estudiantes habían tenido un desempeño nulo. Los estudiantes que habían sido capaces de responder en la prueba diagnóstica esta vez tuvieron un desempeño aceptable, entre tanto que los dos estudiantes que se habían destacado en la prueba previa también mostraron mejoras considerables.

Tabla 9

Sección de speaking

\section{ESTUDIANTES}

\begin{tabular}{llllll}
\hline & $\mathbf{1}$ & $\mathbf{2}$ & $\mathbf{3}$ & $\mathbf{4}$ & $\mathbf{5}$ \\
\hline CLARIDAD & $85 \%$ & $90 \%$ & $55 \%$ & $65 \%$ & $65 \%$ \\
\hline USO DEL LENGUAJE & $90 \%$ & $95 \%$ & $60 \%$ & $60 \%$ & $60 \%$ \\
\hline DESARROLLO DEL TEMA & $90 \% \%$ & $90 \%$ & $55 \%$ & $60 \%$ & $65 \%$
\end{tabular}

Nota. Prueba final de speaking, elaboración propia (2021).

Durante el proceso de este proyecto, el docente investigador estuvo aprendiendo de forma auto didacta acerca de las características de la metodología CLIL, también el investigador se aseguró de recibir formación en el idioma inglés que lo hiciese competente en el nivel B2 del marco común europeo, sin embargo, en el momento previo y durante la puesta en escena de la unidad didáctica se encontró que también es necesario un entrenamiento práctico para el docente en el uso de esta metodología.

Por último, el objetivo de CLIL es que una asignatura no lingüística sea enseñada a través de un idioma extranjero, con el propósito de que por lo menos dos docentes, en este caso el de inglés y el de ciencias sociales trabajen en equipo de modo que el primero le prepare el terreno al segundo. Este proyecto fue llevado a cabo por un docente y 
a pesar de esto, los resultados son alentadores, creyendo que esta metodología tendría un impacto positivo a nivel institucional, si se pudiese llevar a cabo un trabajo en equipo a futuro.

\section{Conclusiones}

La unidad didáctica, de ciencias sociales permitió reflexionar respecto a las necesidades y peculiaridades de los estudiantes. Lo primero que se pudo notar, es que, al adaptar los ejes temáticos al contexto de los estudiantes, estos desempeñan un rol protagónico en el aprendizaje ayudando a descubrir y construir el conocimiento, en el caso particular de ciencias sociales, los estudiantes demostraron fascinación $y$ apropiación por el estudio de su territorio. En segundo lugar, cuando se realizó la integración de una materia como sociales, con el idioma inglés, los estudiantes abordaron el idioma desde otra perspectiva, porque su atención ya no estuvo enfocada en la gramática o lingüística, sino en la línea temática de ciencias sociales, esta manera de aprender idiomas despertó el interés de los estudiantes y estuvieron motivados a aprender inglés.

Por otra parte, los estudiantes tuvieron algunas falencias con la habilidad de "listening" en las primeras sesiones, lo que requirió que se fortaleciera esta habilidad, que fue un prerrequisito para avanzar a la producción oral en el contexto de esta investigación, después de esto fue posible continuar con el desarrollo de contenidos integrando el idioma inglés en aquellos aspectos en los que convergieron. No obstante, se hizo necesario realizar sacrificios tanto temáticos como lingüísticos, seleccionando aquellos conceptos básicos, con el fin de involucrar a todos los estudiantes en un aprendizaje activo.

Finalmente, el aprendizaje integrado de contenidos y lengua (CLIL) ofreció una alternativa a la necesidad lingüística de los estudiantes, y, después del análisis pedagógico de las notas de campo y el contraste entre las pruebas diagnósticas y finales, se concluye que la metodología CLIL tuvo una incidencia que favoreció el uso de las habilidades orales de los estudiantes.

\section{Referencias}

Álvarez, A. y Alvarez, V. (2014) Métodos de la investigación educativa. Universidad pedagógica Nacional.

Ball, P., Clegg J. \& Kelly, K. (2015). Putting CLIL into Practice. Oxford University Press.

Bisquerra, R. (2004) Metodología de la investigación educativa. Editorial la muralla.

Chenlu, L. (2015). Application of Communicative Method in EFL Listening and Speaking Class. Studies in Literature and Language, 10 (5), 1-4.

Contreras, O. (2012). Stephen Krashen: sus aportes a la educación bilingüe. Rastros Rostros, 14 (27). https://revistas.ucc.edu.co/index.php/ra/article/vie w/491

Corbin J. \& Strauss A. (2002) Bases de la investigación cualitativa. Técnicas y procedimientos para desarrollar la teoría fundamentada. Universidad de Antioquia

Hernández-Sampieri, R. Fernández-Collado, C. \& BaptistaLucio, P. (2014). Metodología de la investigación. McGraw-Hill.

Ministerio de Educación Nacional de la República Colombiana. (2004). Formar para la ciudadanía... iSi es posible! Ascofade. http://www.mineducacion.gov.co/1621/articles75768_archivo_pdf.pdf

Ministerio de Educación Nacional República de Colombia. (2006). Formar en Lenguas extranjeras: El Reto, lo que necesitamos saber y saber hacer. Espantapájaros Taller.

Howatt, APR. (1984). A History of English Language Teaching. Oxford University Press.

Marín, J. (2018) Investigar en educación y pedagogía, sus fundamentos epistemológicos. http://bibliotecadigital.magisterio.co.unipamplona. basesdedatosezproxy.com/libro/investigar-eneducaci-n-y-pedagog-sus-fundamentos-epistemolgicos-y-metodol-gicos

Marsh, D. (2002). Clil/Emile: The European Dimension, Action, Trends and Foreignt Potential. http://europa.eu.int/comm/education/lan guages/index.html

Ministerio de educación (2019). Encuentro regional saber, ICFES.

Nunan, D. (2004). Task-Based Language Teaching. Cambridge Language Teaching Library.

PEI (2012). Proyecto Educativo Institucional. Institución educativa José Odel Lizarazo. 
Tamayo-Tamayo, M. (2004). El proceso de la investigación científica-Limusa. Noriega Editores. 